Theoretical Informatics and Applications

Theoret. Informatics Appl. 35 (2001) 403-417

\title{
ON GRAPH PRODUCTS OF AUTOMATIC MONOIDS
}

\author{
A. Veloso DA Costa ${ }^{*, 1}$
}

\begin{abstract}
The graph product is an operator mixing direct and free products. It is already known that free products and direct products of automatic monoids are automatic. The main aim of this paper is to prove that graph products of automatic monoids of finite geometric type are still automatic. A similar result for prefix-automatic monoids is established.
\end{abstract}

Mathematics Subject Classification. 20M10, 68Q68.

\section{INTRODUCTION}

Automatic semigroups were introduced in [1] as an extension of the (automatatheoretic) notion of automatic group. Automatic groups admit two different characterizations: in terms of automata and rational languages or in terms of the geometry of the Cayley graph. A geometric characterization for automatic semigroups (or monoids) has not yet been found. In [7], prefix-automatic monoids are introduced, an apparently stronger definition of automatic monoid. Both definitions coincide for groups but the question is still open for monoids. It is also obtained a geometric characterization of prefix-automatic monoids of finite geometric type (FGT).

Graph products were introduced in [3]. Free products and direct products of monoids are particular cases of graph products. In [1], it is proved that free products and direct products of automatic monoids are automatic and corresponding results for prefix-automatic monoids are proved in [8]. In [5], it is proved that the graph product of automatic groups is automatic. We prove, in Theorem 6.4 , that the graph product of automatic monoids of finite geometric type is automatic.

\footnotetext{
* Supported by PRODEP II.

Keywords and phrases: Automatic monoid, graph product.

1 Centro de Matemática, Universidade do Minho, 4710-057 Braga, Portugal;

e-mail: aveloso@math.uminho.pt
}

(c) EDP Sciences 2002 
The corresponding result for prefix-automatic monoids is established in Theorem 6.5. We also prove that the graph product of FGT monoids is FGT.

\section{GRAph PRODUCTS OF MONOIDS}

For more details on this section the reader is referred to [9]. Let $X$ be a nonempty set. As usual, the free monoid on $X$ is denoted by $X^{*}$. If $R$ is a relation on $X^{*}$, the congruence on $M$ generated by $R$ is denoted by $R^{\sharp}[6]$ and the formal expression $\langle X ; R\rangle$ is said to be a presentation. The quotient $X^{*} / R^{\sharp}$ is said to be the monoid defined by the presentation $\langle X ; R\rangle$. Given a monoid $M$, we say that $\langle X ; R\rangle$ is a presentation of $M$ if $X^{*} / R^{\sharp}$ is isomorphic to $M$.

The graph product is an operator mixing direct and free products. Whether the product between two monoids is free or direct is determined by a simplicial graph, that is, a (non directed) graph with no loops. Considering a monoid attached to each vertex of the graph, the associated graph product is the monoid generated by each of the vertex monoids with the added relations that elements of adjacent vertex monoids commute. Formally, given a finite family of monoids $M_{1}, M_{2}, \ldots, M_{n}$ with presentations $\left\langle X_{1} ; R_{1}\right\rangle,\left\langle X_{2} ; R_{2}\right\rangle, \ldots,\left\langle X_{n} ; R_{n}\right\rangle$ (we assume that the sets $X_{1}, X_{2}, \ldots, X_{n}$ are disjoint) and $\Gamma$ a simplicial graph with vertices labeled $1,2, \ldots, n$, the associated graph product is a monoid $\Gamma\left(M_{1}, M_{2}, \ldots, M_{n}\right)$ with presentation $\langle X ; R\rangle$, where

$$
X=\bigcup_{i=1}^{n} X_{i}, \quad R=\bigcup_{i=1}^{n} R_{i} \cup R_{\Gamma}
$$

and

$$
R_{\Gamma}=\left\{(a b, b a): a \in X_{i}, b \in X_{j} \text { and } i, j \text { are adjacent vertices of } \Gamma\right\}
$$

Two elements $a$ and $b$ in $X$ are said to $\Gamma$-commute $(a \sim b)$ if they arise from adjacent monoids. The same notation is used for monoids $\left(M_{i} \sim M_{j}\right)$ and indices $(i \sim j)$.

Given a word $u$ in $X^{*}$, we represent the $n$-th letter of $u$ by $u(n)$ and the length of $u$ by $|u|$. We say that $v \in X^{*}$ is a subword of $u$ if $v=u\left(i_{1}\right) u\left(i_{2}\right) \cdots u\left(i_{k}\right)$, where $1 \leq i_{1}<i_{2}<\cdots<i_{k} \leq|u|$. Let $u \in X^{*}$ and $i \in\{1,2, \cdots, n\}$. An $i$-component of $u$ (or a component of type $i$ ) is a maximal subword of $u, v=v_{1} v_{2} \cdots v_{k}$ such that, for every $j \in\{1,2, \cdots, k\}, v_{j} \in X_{i}$ and (for $j \neq k$ ) every symbol occurring in $u$ between $v_{j}$ and $v_{j+1} \Gamma$-commutes with $i$. The first element of a component of $u$ is said to be the head element of the component. We define $S(u)$ as the product of the components of $u$ ordered by their head elements. Let $S\left(X^{*}\right)=\left\{S(u): u \in X^{*}\right\}$. Notice that, given a word $u$ in $X^{*}, S(u)$ and $u$ are congruent in $M$. For words in $S\left(X^{*}\right)$ we usually write the components between square brackets to emphasize them. We define the type of a word in $S\left(X^{*}\right)$ as the sequence of types of its components. 
Let $u, v \in S\left(X^{*}\right)$. We say that:

$u$ is $N$-equivalent to $v\left(u \Leftrightarrow_{N} v\right)$ : if $u$ and $v$ have the same type and the components in the same position are congruent in the respective monoid.

$v$ results from $u$ by a $\Gamma$-transition $\left(u \leftrightarrow_{\Gamma} v\right)$ : if $v$ results from $u$ by swapping two consecutive components that $\Gamma$-commute.

$u$ is $\Gamma$-equivalent to $v\left(u \Leftrightarrow_{\Gamma} v\right)$ : if $v$ results from $u$ by a finite number of $\Gamma$-transitions.

$u$ is $\Gamma_{N}$-equivalent to $v\left(u \Leftrightarrow_{\Gamma_{N}} v\right)$ : if there is $w$ in $S\left(X^{*}\right)$ such that $u \Leftrightarrow_{\Gamma}$ $w$ and $w \Leftrightarrow_{N} v$.

If $u \Leftrightarrow \Gamma_{N} v$, since $\Gamma$-transitions do not commute components of the same type, we have that, for all $n$ and $i$ the $n$-th $i$-components of $u$ and $v$ are congruent in $M_{i}$. So, if $u \Leftrightarrow \Gamma_{N} v$ we have a natural notion of corresponding components in $u$ and $v$. Notice that $\Leftrightarrow_{N} \subseteq \Leftrightarrow_{\Gamma_{N}}$ and $\Leftrightarrow_{\Gamma} \subseteq \Leftrightarrow_{\Gamma_{N}}$. Since both relations are also contained in $R^{\sharp}$ the relation $\Leftrightarrow_{\Gamma_{N}}$ is contained in $R^{\sharp}$ too. The relations $\Leftrightarrow_{N}, \Leftrightarrow_{\Gamma}$ and $\Leftrightarrow_{\Gamma_{N}}$ are equivalence relations on $S\left(X^{*}\right)$.

Let $u$ be a word in $S\left(X^{*}\right)$ and $[t]$ one of its components. We say that $[t]$ is initial (terminal) for $u$ if $[t] \Gamma$-commutes with all the components of $u$ on the left (right) of $[t]$. Any word $u \in S\left(X^{*}\right)$ such that its components are of different types and all $\Gamma$-commute will be called a block. Notice that given $u \in S\left(X^{*}\right)$ any two initial (terminal) components for $u \Gamma$-commute. Moreover, we can rewrite $u$, by $\Gamma$-transitions, in such a way that all the initial (terminal) components are in the beginning (end) of the word, forming a block.

Given $u \in S\left(X^{*}\right)$, when we suppress a trivial component, that is, a component that is congruent to the empty word in the corresponding monoid, we get a new word that is congruent to $u$ in $M$. After this we want to write the resulting word as a product of its components again. We will call that process a reduction of $u$. Any word that admits no reductions will be called an irreducible word. Given a word $u$ in $S\left(X^{*}\right)$, we will call irreducible form of $u$ any irreducible word obtained from $u$ by a (finite) sequence of reductions. Now we list some results obtained in $[9]$.

Lemma 2.1 ([9], Lem. 4.1). Let $u, v \in S\left(X^{*}\right)$ be $\Gamma_{N}$-equivalent. If $[t]$ is a component of $u$ and $[s]$ is the corresponding component of $v$, then $[t]$ is initial (terminal) for $u$ if and only if $[s]$ is initial (terminal) for $v$.

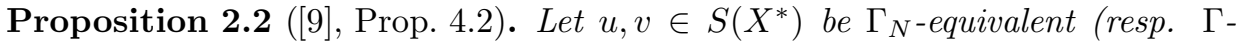
equivalent, $N$-equivalent). If $[t]$ is an initial or terminal component of $u$ and $[s]$ is the corresponding component of $v$ then cutting $[t]$ in $u$ and $[s]$ in $v$ we get words in $S\left(X^{*}\right)$ that are $\Gamma_{N}$-equivalent (resp. $\Gamma$-equivalent, $N$-equivalent).

Theorem 2.3 ([9], Th. 6.1). Let $u, v \in X^{*}$. The following conditions are equivalent:

1. $u$ and $v$ are congruent in $M$;

2. any irreducible forms of $S(u)$ and $S(v)$ are $\Gamma_{N}$-equivalent;

3. there are irreducible forms of $S(u)$ and $S(v)$ that are $\Gamma_{N}$-equivalent. 


\section{Rational Languages AND AUtomata}

Let $A$ be a finite alphabet. As usual, $A^{+}$denotes the set of all non-empty words over $A$ and $A^{*}$ denotes the set of all words over $A$, including the empty word represented by 1 . A language over the alphabet $A$ is a subset of $A^{*}$. For every word $u$ over an alphabet $A$, let $|u|$ denote the length of $u$ and for every integer $k$ such that $0 \leq k \leq|u|$, let $u(k)$ denote the prefix of $u$ with length $k$. Given $L \subseteq A^{*}$, we denote by $\operatorname{Pref}(L)$ the set of all prefixes of words in $L$.

A finite automaton is a 5 -tuple $\mathcal{A}=\left(Q, A, \mu, q_{0}, F\right)$ where $Q$ and $A$ are finite sets (called the states and input alphabet, respectively), $\mu$ is a partial function from $Q \times A$ to $Q, q_{0} \in Q$ is the initial state and $F \subseteq Q$ is the set of final states. The interpretation of $(q, a) \mu=q^{\prime}$ is that $\mathcal{A}$ in state $q$ with input symbol a moves to state $q^{\prime}$. We extend $\mu$ to $Q \times A^{*}$ as follows:

1) for every $q \in Q,(q, 1) \mu=q$;

2) for every $u \in A^{*}$ and $a \in A$, we define $(q, u a) \mu=((q, u) \mu, a) \mu$ if $(q, u) \mu$ and $((q, u) \mu, a) \mu$ are defined.

A word $u \in A^{*}$ is said to be accepted or recognized by $\mathcal{A}$ if $\left(q_{0}, u\right) \mu$ is defined and $\left(q_{0}, u\right) \mu \in F$. The set of words in $A^{*}$ that are accepted by $\mathcal{A}$ is said to be the language accepted or recognized by $\mathcal{A}$. A language over $A$ is said to be rational if it is recognized by a finite automaton over $A$. We will now list some well-known properties of rational languages (see [4]).

Lemma 3.1. Assume that $A$ and $B$ are finite sets. Then:

(i) $\emptyset, A^{+}$and $A^{*}$ are rational;

(ii) any finite subset of $A^{*}$ is rational;

(iii) if $K, L \subseteq A^{*}$ are rational then $K L, K \cup L, K \cap L, K-L$ and $K^{*}$ are rational;

(iv) If $K \subseteq A^{*}$ is rational and $\Phi: A^{*} \rightarrow B^{*}$ is a monoid homomorphism, then $K \Phi$ is rational;

(v) If $K \subseteq B^{*}$ is rational and $\Phi: A^{*} \rightarrow B^{*}$ is a monoid homomorphism, then $K \Phi^{-1}$ is rational.

We will now introduce a generalization of the notion of finite automaton.

A generalized sequential machine $(\mathrm{GSM})$ is a 6 -tuple $\mathcal{M}=\left(Q, A, B, \mu, q_{0}, F\right)$ where $Q, A$ and $B$ are finite sets (called the states, input alphabet and output alphabet, respectively), $\mu$ is a function from $Q \times A$ to finite subsets of $Q \times B^{*}$, $q_{0} \in Q$ is the initial state and $F \subseteq Q$ is the set of final states. The interpretation of $\left(q^{\prime}, w\right) \in(q, a) \mu$ is that $\mathcal{M}$ in state $q$ with input symbol $a$ may, as one possible choice of move, enter state $q^{\prime}$ and emit string $w$. We extend $\mu$ to $Q \times A^{*}$ as follows:

1) for every $q \in Q,(q, 1) \mu=\{(q, 1)\}$;

2) for every $u \in A^{*}$ and $a \in A,(q, u a) \mu=$

$\left\{\left(q_{2}, w\right) \mu: w=w_{1} w_{2}\right.$ and for some $q_{1},\left(q_{1}, w_{1}\right) \in(q, u) \mu$ and $\left.\left(q_{2}, w_{2}\right) \in\left(q_{1}, a\right) \mu\right\}$.

For every $u \in A^{*}$ let $\mathcal{M}(u)=\left\{w:\left(q^{\prime}, w\right) \in\left(q_{0}, u\right) \mu\right.$, for some $\left.q^{\prime} \in F\right\}$ and for every $L \subseteq A^{*}$ let $\mathcal{M}(L)=\{w: w \in \mathcal{M}(u)$ for some $u \in L\}$. We can see $\mathcal{M}$ as a map from the set of all languages over $A$ to the set of all languages over $B$. 
Moreover, if $L$ is a rational language over $A$ then $\mathcal{M}(L)$ is a rational language over $B$ ([4], Th. 11.1).

Assume now that $\$$ is a symbol not in $A$. We define

$$
A(2, \$)=(A \cup\{\$\}) \times(A \cup\{\$\})-\{(\$, \$)\}
$$

and we define a mapping $\delta: A^{*} \times A^{*} \rightarrow A(2, \$)^{*}$ as follows. Given $u=a_{1} \cdots a_{n}$ and $v=a_{1}^{\prime} \cdots a_{m}^{\prime}$, with $a_{i}, a_{j}^{\prime} \in A$, let

$$
(u, v) \delta= \begin{cases}1 & \text { if } n=m=0 \\ \left(a_{1}, a_{1}^{\prime}\right) \cdots\left(a_{n}, a_{n}^{\prime}\right) & \text { if } n=m>0 \\ \left(a_{1}, a_{1}^{\prime}\right) \cdots\left(a_{n}, a_{n}^{\prime}\right)\left(\$, a_{n+1}^{\prime}\right) \cdots\left(\$, a_{m}^{\prime}\right) & \text { if } 0 \leq n<m \\ \left(a_{1}, a_{1}^{\prime}\right) \cdots\left(a_{m}, a_{m}^{\prime}\right)\left(a_{m+1}, \$\right) \cdots\left(a_{n}, \$\right) & \text { if } n>m \geq 0 .\end{cases}
$$

The diagonal homomorphism $\Delta: A^{*} \rightarrow A(2, \$)^{*}$ is defined by $a \Delta=(a, a)$, for $a \in A$. Let $\pi_{1}$ and $\pi_{2}$ be the natural projections from $((A \cup\{\$\}) \times(A \cup\{\$\}))^{*}$ into $(A \cup\{\$\})^{*}$ defined by $\left(a_{1}, a_{2}\right) \pi_{1}=a_{1}$ and $\left(a_{1}, a_{2}\right) \pi_{2}=a_{2}$ for every $a_{1}, a_{2} \in A \cup\{\$\}$. For every $u \in(A \cup\{\$\})^{*}$ let $\#_{\$}(u)$ be the number of occurrences of $\$$ in $u$. For every $u \in A(2, \$)^{*}$ let $\sigma(u)=\left|\#_{\$}\left(u \pi_{1}\right)-\#_{\$}\left(u \pi_{2}\right)\right|$ and let $\Sigma(u)=\operatorname{Max}\{\sigma(u(k))$ : $1 \leq k \leq|u|\}$. For a language $L \subseteq A(2, \$)^{*}$ we define $\Sigma(L)=\operatorname{Sup}\{\Sigma(u): u \in L\}$.

Consider now the homomorphism $\alpha:(A \cup\{\$\})^{*} \rightarrow A^{*}$ defined by $a \alpha=a$, if $a \in A$, and $\$ \alpha=1$. Let $\zeta: A(2, \$)^{*} \rightarrow\left(A^{*} \times A^{*}\right) \delta$ defined by $u \zeta=\left(u \pi_{1} \alpha, u \pi_{2} \alpha\right) \delta$.

Lemma 3.2. If $L \subseteq A(2, \$)^{*}$ is a rational language and $\Sigma(L)$ is finite then $L \zeta$ is rational.

Proof. This is a technical lemma. For readers familiarized with GSMs, the proof is straightforward. We include here a detailed proof.

If $\Sigma(L)=0$ then $L \zeta=L$ and the result holds. Assume then that $\Sigma(L)>0$. We will construct a GSM $\mathcal{M}$ such that $\mathcal{M}(L)=L \zeta$.

We take $A(2, \$)$ for both input and output alphabets. Let $Q_{1}$ be the set of all words in $(A \times\{\$\})^{+}$with length less or equal to $\Sigma(L)$ and let $Q_{2}$ be the set of all words in $(\{\$\} \times A)^{+}$with length less or equal to $\Sigma(L)$. Let $Q=Q_{1} \cup Q_{2} \cup\left\{q_{0}, t\right\}$ where $q_{0}$ is the initial state and $t$ is the single final state. Since alphabet $A$ is finite, $Q$ is also finite. Now we will explain how to construct the function $\mu: Q \times A(2, \$) \rightarrow Q \times A(2, \$)^{*}$.

Let $a_{1}, a_{2}, b_{1} \in A$. When at initial state $q_{0}$, if we input $\left(a_{1}, \$\right)$, since $\left(a_{1}, \$\right)$ is not in $A \times A$, we move to state $\left(a_{1}, \$\right)$ and generate 1 as output. We have now three cases to consider: if $\Sigma(L)>1$ and we input $\left(a_{2}, \$\right)$ then we move to state $\left(a_{1}, \$\right)\left(a_{2}, \$\right)$ and output 1 ; if the input is of the form $\left(\$, b_{1}\right)$ then we move to initial state $q_{0}$ and generate $\left(a_{1}, b_{1}\right)$ as output; if we input $\left(a_{2}, b_{1}\right)$ then we move to state $\left(a_{2}, \$\right)$ and output $\left(a_{1}, b_{1}\right)$.

As we read input we generate pairs in $A \times A$ and the "remaining" part of input read is stored in the current state. When input ends we must output the word stored at the current state. This can be done proceeding as for automata: when at 
state $q_{1}$ input $u \in A(2, \$)$ allows a move to state $q_{2}$ producing output $v$ we allow an alternative move from $q_{1}$, with the same input $u$, to the final state $t$ generating as output $v q_{2}$, where here $q_{2}$ is understood as a word. Hence, we formally define $\mu$ as follows:

For all $a, b \in A$,

$$
\begin{aligned}
& \left(q_{0},(a, b)\right) \mu=\left\{\left(q_{0},(a, b)\right),(t,(a, b))\right\} ; \\
& \left(q_{0},(a, \$)\right) \mu=\{((a, \$), 1),(t,(a, \$))\} ; \\
& \left(q_{0},(\$, b)\right) \mu=\{((\$, b), 1),(t,(\$, b))\} .
\end{aligned}
$$

Let $q=\left(a_{1}, \$\right)\left(a_{2}, \$\right) \cdots\left(a_{m}, \$\right) \in Q_{1}$. If $0<m<\Sigma(L)$, we define for all $a, b \in A$,

$$
\begin{gathered}
(q,(a, b)) \mu=\left\{\left(\left(a_{2}, \$\right) \cdots\left(a_{m}, \$\right)(a, \$),\left(a_{1}, b\right)\right),\left(t,\left(a_{1}, b\right)\left(a_{2}, \$\right) \cdots\left(a_{m}, \$\right)(a, \$)\right)\right\} \\
(q,(a, \$)) \mu=\left\{\left(\left(a_{1}, \$\right)\left(a_{2}, \$\right) \cdots\left(a_{m}, \$\right)(a, \$), 1\right),\left(t,\left(a_{1}, \$\right)\left(a_{2}, \$\right) \cdots\left(a_{m}, \$\right)(a, \$)\right)\right\} \\
(q,(\$, b)) \mu=\left\{\left(\left(a_{2}, \$\right) \cdots\left(a_{m}, \$\right),\left(a_{1}, b\right)\right),\left(t,\left(a_{1}, b\right)\left(a_{2}, \$\right) \cdots\left(a_{m}, \$\right)\right)\right\}
\end{gathered}
$$

If $m=\Sigma(L)$, for all $a, b \in A$, we define $(q,(a, b)) \mu$ and $(q,(\$, b)) \mu$ in the same way and we define

$$
(q,(a, \$)) \mu=\emptyset .
$$

For states in $Q_{2}, \mu$ is defined in a similar way and, for state $t$, we define, for all $a, b \in A$,

$$
(t,(a, b)) \mu=(t,(a, \$)) \mu=(t,(\$, b)) \mu=\emptyset .
$$

Since $t$ is the single final state of $\mathcal{M}$, for every $u \in L, \mathcal{M}(u)=\{w:(t, w) \in$ $\left.\left(q_{0}, u\right) \mu\right\}$. At every state $q \neq t$ any input in $A(2, \$)$ is accepted (with exception of the states with length $\Sigma(L))$ and we always have two optional moves where one of them is a move to the final state $t$. On the other hand, since at state $t$ no move is allowed for any input, the option for a move to state $t$ should be taken only in the final move. Let $u \in L$. Since no prefix $v$ of $u$ satisfies $\sigma(v)>\Sigma(L)$ we conclude that there is exactly one path from $q_{0}$ to $t$ labeled by $u$ and thus $\mathcal{M}(u)$ has a single element. Assume that $\mathcal{M}(u)=\{w\}$. To see that $w=u \zeta$ we just have to note the following:

- with the exception of the final move, every output generated is in $A \times A$ and thus $w \in\left(A^{*} \times A^{*}\right) \delta$;

- input symbols of $u \pi_{1} \alpha$ are outputed by the same order and no other symbol of $A$ occurs in $w \pi_{1} \alpha$. Hence $u \pi_{1} \alpha=w \pi_{1} \alpha$;

- input symbols of $u \pi_{2} \alpha$ are outputed by the same order and no other symbol of $A$ occurs in $w \pi_{2} \alpha$. Hence $u \pi_{2} \alpha=w \pi_{2} \alpha$.

Hence we must have

$$
w=\left(w \pi_{1} \alpha, w \pi_{2} \alpha\right)=\left(u \pi_{1} \alpha, u \pi_{2} \alpha\right) \delta=u \zeta .
$$

Thus, $\mathcal{M}(L)=L \zeta$ and therefore $L \zeta$ is rational. 


\section{Automatic monoids}

Let $M$ be a monoid and let $\phi: A^{+} \rightarrow M$ be a surjective semigroup homomorphism. We say that $L \subseteq A^{*}$ is a rational section for $\phi$ if $L$ is rational and $\left.\phi\right|_{L}: L \rightarrow M$ is surjective (where we extend $\phi$ to $A^{*}$ in the usual manner). If, in addition, $\left.\phi\right|_{L}$ is injective, $L$ is called a rational cross-section for $\phi$. Given a rational section $L$ for $\phi$ and $w \in A^{*}$, we define

$$
\begin{gathered}
L_{w}=\{(u, v) \delta: u, v \in L \text { and } v \phi=(u w) \phi\} ; \\
L_{=}^{\prime}=\{(u, v) \delta: u \in L, v \in \operatorname{Pref}(L) \text { and } u \phi=v \phi\} .
\end{gathered}
$$

The language $L_{1}$ will also be denoted $L_{=}$.

A rational section $L$ is said to be an automatic structure for $\phi$ if, [1]

(i) $L_{a}$ is rational for every $a \in A$;

(ii) $L_{=}$is rational.

If, in addition,

(iii) $L_{=}^{\prime}$ is rational,

then $L$ is said to be a prefix-automatic or p-automatic structure for $\phi$ [7]. If $L$ is a rational cross-section then condition (ii) is redundant and we say that $L$ is a (p-)automatic structure with uniqueness for $\phi$.

A monoid $M$ is said to be ( $p$-)automatic if there is a finite alphabet $A$ and a surjective homomorphism $\phi: A^{+} \rightarrow M$ with an (p-)automatic structure. It would be more natural to define (p-)automatic monoids using monoid homomorphisms $\phi: A^{*} \rightarrow M$. Both definitions are equivalent but with the definition used here automaticity is independent from the generating set considered [2].

\section{Monoids OF Finite GEOMETRIC TYPE}

A monoid $M$ is said to be of finite geometric type (FGT) if it is finitely generated and, for every $p \in M$, there is an integer $m$ such that the equation $x p=q$ has at most $m$ solutions for every $q \in M$. The choice of terminology is more evident when we consider the following alternative characterization of FGT monoids:

Proposition 5.1 ([7], Prop. 2.1). Given a finitely generated monoid $M$, the following conditions are equivalent:

i) $M$ is $F G T$;

ii) Given a finite set $A$ and a surjective semigroup homomorphism $\phi: A^{+} \rightarrow M$, there is an integer $m$ such that there are at most $m$ coterminal edges with the same label in the Cayley graph with respect to A.

Groups and right cancellative monoids are FGT. On the other hand, monoids with zero are examples of non-FGT monoids. To prove that the graph product of FGT 
automatic monoids is automatic we will need the following:

Lemma 5.2. Let $M$ be an automatic monoid of finite geometric type. Let $A$ be a finite set, $\phi: A^{+} \rightarrow M$ a surjective semigroup homomorphism and $L \subseteq A^{*}$ an automatic structure with uniqueness for $\phi$. Then, for every $a \in A, \Sigma\left(L_{a}\right)$ is finite.

Proof. Let $a \in A$. Since $L_{a}$ is rational, then $L_{a}$ is recognized by a finite automaton $\mathcal{A}$. Assume that $\mathcal{A}$ has $k_{a}$ states and let $u, v \in L$ be such that $v \phi=(u a) \phi$. We will use a variant of the Pumping Lemma for rational languages ([4], Lem. 3.1).

If $|u|<|v|$ then $|v|-|u| \leq k_{a}$ otherwise there would be a cycle in $\mathcal{A}$ labeled by $\left(1, v^{\prime}\right) \delta$, for some non-empty factor $v^{\prime}$ of $v$. Then, assuming that $v=v_{1} v^{\prime} v_{2}$, for every integer $n$ we would have $\left(u, v_{1}\left(v^{\prime}\right)^{n} v_{2}\right) \delta \in L_{a}$. Hence, for every $n$, we would have $v_{1}\left(v^{\prime}\right)^{n} v_{2} \in L$ and $\left(v_{1}\left(v^{\prime}\right)^{n} v_{2}\right) \phi=v \phi$, contradicting $L$ being an automatic structure with uniqueness.

If $|u|>|v|$ then $|u|-|v| \leq k_{a}$ otherwise there would be a cycle in $\mathcal{A}$ labeled by $\left(u^{\prime}, 1\right) \delta$, for some non-empty factor $u^{\prime}$ of $u$. Then, assuming that $u=u_{1} u^{\prime} u_{2}$, for every integer $n$ we would have $\left(u_{1}\left(u^{\prime}\right)^{n} u_{2}, v\right) \delta \in L_{a}$. Hence, for every $n$, we would have $u_{1}\left(u^{\prime}\right)^{n} u_{2} \in L$ and $\left(u_{1}\left(u^{\prime}\right)^{n} u_{2}\right) \phi(a \phi)=v \phi$, a contradiction since $M$ is of finite geometric type and $L$ is an automatic structure with uniqueness.

Hence, $\Sigma((u, v) \delta)=\|u|-| v\| \leq k_{a}$ and therefore $\Sigma\left(L_{a}\right)$ is finite.

\section{Graph products of automatic monoids}

Free products and direct products of monoids are particular cases of graph products of monoids. In fact, if the graph $\Gamma$ is totally disconnected then $\Gamma\left(M_{1}, M_{2}, \ldots\right.$, $\left.M_{n}\right)=M_{1} * M_{2} * \cdots * M_{n}$ and if $\Gamma$ is a complete graph then $\Gamma\left(M_{1}, M_{2}, \ldots, M_{n}\right)$ is isomorphic to $M_{1} \times M_{2} \times \cdots \times M_{n}$ (note that is not the case for semigroups). It is already known that automaticity for monoids is preserved by free products ([1], Th. 6.2) and direct products ([1], Th. 6.4). These proofs cannot be immediately generalized for graph products. We will see that graph products preserve automaticity if monoids $M_{i}$ are of finite geometric type.

Let $M_{1}, M_{2}, \ldots, M_{n}$ be automatic monoids and let $M=\Gamma\left(M_{1}, M_{2}, \ldots, M_{n}\right)$ be the graph product of such monoids associated to a graph $\Gamma$. Then, adapting $([1]$, Cor. 5.6, Prop. 5.7 and Prop. 5.3) for the monoid case, we may assume that there are automatic structures with uniqueness $L_{i}$ for $\phi_{i}: A_{i}^{+} \rightarrow M_{i}$ such that $1 \in L_{i}$, for every $i$. Furthermore, we may assume that the generating sets $A_{i}$ are disjoint. Let $A$ be the union of the sets $A_{i}$ and let $\phi: A^{+} \rightarrow M$ be the semigroup homomorphism defined by $a \phi=a \phi_{i} \theta_{i}$, if $a \in A_{i}$, where $\theta_{i}$ is the natural embedding of $M_{i}$ into $M$. Since $M$ is generated by the submonoids $M_{i} \theta_{i}, \phi$ is surjective. We want to construct a rational cross-section for $\phi$. For that we will have to introduce more notation.

For every $i$, let $\overline{L_{i}}=L_{i}-\{1\}$. Given a subset $\left\{i_{1}, i_{2}, \cdots, i_{k}\right\}$ of $\{1,2, \cdots, n\}$ we say that $\left\{i_{1}, i_{2}, \cdots, i_{k}\right\}$ is independent if the subgraph of $\Gamma$ induced by vertices $i_{1}, i_{2}, \cdots, i_{k}$ is complete. Let $\mathcal{I}$ be the set of all independent subsets of $\{1,2, \cdots, n\}$. A non-empty sequence $\left(i_{1}, i_{2}, \cdots, i_{k}\right)$ such that $1 \leq i_{1}<i_{2}<$ 
$\cdots<i_{k} \leq n$ and $\left\{i_{1}, i_{2}, \cdots, i_{k}\right\}$ is independent is said to be an independent sequence. For every $j \in\{1,2, \cdots, n\}$ we say that an independent sequence $\left(i_{1}, i_{2}, \cdots, i_{k}\right) \Gamma$-commutes with $j\left(\left(i_{1}, i_{2}, \cdots, i_{k}\right) \sim j\right)$ if $j \notin\left\{i_{1}, i_{2}, \cdots, i_{k}\right\}$ and $\left\{i_{1}, i_{2}, \cdots, i_{k}\right\} \cup\{j\} \in \mathcal{I}$. For every independent sequence $\left(i_{1}, i_{2}, \cdots, i_{k}\right)$ let

$$
B_{i_{1}, i_{2}, \cdots, i_{k}}=\overline{L_{i_{1}}} \overline{L_{i_{2}}} \cdots \overline{L_{i_{k}}}
$$

We say that $B_{i_{1}, i_{2}, \cdots, i_{k}}$ is a block language of type $\left(i_{1}, i_{2}, \cdots, i_{k}\right)$. Any terminology introduced for independent sequences or block languages will be used for both concepts. A finite product of block languages is said to be admissible if for any two consecutive block languages $B_{i_{1}, i_{2}, \cdots, i_{k}}$ and $B_{j_{1}, j_{2}, \cdots, j_{l}}$ we have

(i) $\left\{i_{1}, i_{2}, \cdots, i_{k}\right\} \cap\left\{j_{1}, j_{2}, \cdots, j_{l}\right\}=\emptyset$;

(ii) for every $p \in\left\{j_{1}, j_{2}, \cdots, j_{l}\right\}$ there is $q \in\left\{i_{1}, i_{2}, \cdots, i_{k}\right\}$ such that $p \nsim q$.

Let $L$ be the union of all admissible (finite) products of block languages together with the empty word. This construction of $L$ is inspired on the work developed in [9] and we will use that work to prove that $L$ is a rational cross-section for $\phi$.

Proposition 6.1. $L$ is a cross-section for $\phi$.

Proof. Let $x \in M$ and let $u \in A^{+}$be such that $u \phi=x$. Now consider any irreducible form of $u$ and, for every $i$, replace the $i$-components by the corresponding words of $\overline{L_{i}}$. Then, write the initial components, ordered by their types, at the beginning of the word and proceed in the same way for the remaining part of the word. The word obtained by this process is in $L$ and is congruent to $u$. Hence $\left.\phi\right|_{L}: L \rightarrow M$ is surjective (where we extend $\phi$ to $A^{*}$ in the usual manner).

Assume now that $u, v \in L$ are such that $u \phi=v \phi$. Then $u$ and $v$ are irreducible words and, by Theorem 2.3 , they are $\Gamma_{N}$-equivalent. Let $\left[u_{i}\right]$ and $\left[v_{i}\right]$ be corresponding $i$-components of $u$ and $v$, respectively. Then $\left[u_{i}\right]$ and $\left[v_{i}\right]$ are congruent in $M_{i}$ and since $L_{i}$ is a cross-section for $\phi_{i}$, they are equal. Hence $u$ and $v$ are $\Gamma$-equivalent. Then, by construction of $L$ and using successively Lemma 2.1 and Proposition 2.2, we conclude that $u=v$.

\section{Proposition 6.2. L is rational.}

Proof. Since, for every $i$, the languages $L_{i}$ are rational then, by Lemma 3.1, the languages $\overline{L_{i}}$ are rational. Hence, again by Lemma 3.1, any block language is rational. A language over an alphabet $A$ is rational if it is accepted by a finite automaton over $A$. Moreover, if we have a rational language and we substitute any symbol of $A$ by a rational language the resulting language is still rational (see [4], Th. 3.4). Hence we can prove that $L$ is rational constructing a finite automaton where the alphabet is the set of block languages and the language accepted by the automaton is the set of admissible products of block languages together with the empty word.

Let $B$ be the set of all block languages. Notice that, since $\Gamma$ is finite, $B$ is also finite. Let $Q=B \cup\left\{q_{0}\right\}$ be the set of states of the automaton where $q_{0}$ is the 
initial state. Take $B$ for alphabet and define $\mu$ as follows:

- for the initial state $q_{0}$ any input $b \in B$ is accepted and $\left(q_{0}, b\right) \mu=b$;

- for any other state $b$ input $b^{\prime}$ is accepted if $b b^{\prime}$ is an admissible product and then $\left(b, b^{\prime}\right) \mu=b^{\prime}$.

Taking $Q$ for set of final states, the language accepted by this automaton is $L$ and thus $L$ is rational.

Since $L$ is a rational cross-section for $\phi$ then $L=L \Delta$ and thus, by Lemma 3.1, $L=$ is rational.

Let $a \in A_{i}$ for some $i \in\{1,2, \cdots, n\}$. We want to prove that $L_{a}$ is a rational language. If $a \phi_{i}=1$ then for every $u \in L,(u a) \phi=u \phi$ and thus $L_{a}=L \Delta$ is rational. From now on we will assume that $a \phi_{i} \neq 1$.

For every independent sequence $\left(i_{1}, i_{2}, \cdots, i_{k}\right)$ let $X_{i_{1}, i_{2}, \cdots, i_{k}}$ be the union of all admissible products $P$ of block languages such that $P B_{i_{1}, i_{2}, \cdots, i_{k}}$ is still admissible, together with the empty word 1 . Let $T_{i_{1}, i_{2}, \cdots, i_{k}}=X_{i_{1}, i_{2}, \cdots, i_{k}} B_{i_{1}, i_{2}, \cdots, i_{k}}$.

For every independent sequence $\left(i_{1}, i_{2}, \cdots, i_{k}\right)$ and for every $i \in\{1,2, \cdots, n\}$ such that $\left(i_{1}, i_{2}, \cdots, i_{k}\right) \sim i$ let $Y_{i_{1}, i_{2}, \cdots, i_{k}}^{i}$ be the union of all admissible products $P$ of block languages such that $B_{i_{1}, i_{2}, \ldots, i_{k}} P$ is still admissible and each block language in $P \Gamma$-commutes with $i$, together with the empty word 1 . Let $I_{i_{1}, i_{2}, \cdots, i_{k}}^{i}=B_{i_{1}, i_{2}, \cdots, i_{k}} Y_{i_{1}, i_{2}, \cdots, i_{k}}^{i}$.

Proposition 6.3. Let $\left(i_{1}, i_{2}, \cdots, i_{k}\right)$ be an independent sequence. Then

(i) $X_{i_{1}, i_{2}, \cdots, i_{k}}$ is rational;

(ii) if $i \in\{1,2, \cdots, n\}$ is such that $\left(i_{1}, i_{2}, \cdots, i_{k}\right) \sim i$ then $Y_{i_{1}, i_{2}, \cdots, i_{k}}^{i}$ is rational.

Proof. Let $\mathcal{A}$ be the automaton considered in the proof of Proposition 6.2. Now take for new set of final states all states that accept a move to $B_{i_{1}, i_{2}, \cdots, i_{k}}$. The language accepted by the new automaton is $X_{i_{1}, i_{2}, \cdots, i_{k}}$.

If there is $i \in\{1,2, \cdots, n\}$ such that $\left(i_{1}, i_{2}, \cdots, i_{k}\right) \sim i$, let $\mathcal{A}^{\prime}=\left(Q^{\prime}, B, \mu^{\prime}, q_{0}^{\prime}\right.$, $\left.F^{\prime}\right)$ be an automaton over $B$, the set of all block languages, where: $Q^{\prime}=\{q \in$ $\left.Q-\left\{q_{0}\right\}: q \sim i\right\}, \mu^{\prime}=\left.\mu\right|_{Q^{\prime} \times B}, q_{0}^{\prime}=B_{i_{1}, i_{2}, \cdots, i_{k}}$ and $F^{\prime}=Q^{\prime}$. Then the language accepted by $\mathcal{A}^{\prime}$ is $Y_{i_{1}, i_{2}, \cdots, i_{k}}^{i}$.

Let $u$ be a word in $L$. We want to find the element $v \in L$ such that $v \phi=(u a) \phi$. We know that if $u \neq 1, u$ belongs to some admissible product of block languages $B_{1} B_{2} \cdots B_{m}$ and then we may assume that $u=u_{1} u_{2} \cdots u_{m}$ where $u_{j} \in B_{j}$, for every $j \in\{1,2, \cdots, m\}$. If $B_{m} \sim i$ then swapping $a$ and $u_{m}$ we obtain a new word that represents the same element in $M$. Proceeding in the same way for the following words $u_{m-1}, u_{m-2}, \cdots$, we will reach (if it exists) a word $u_{j} \in B_{j}$ such that $B_{j} \nsim i$. This procedure induces a unique factorization in $u$ and $B_{1} B_{2} \cdots B_{m}$. Then given $u \in L$ and $i \in\{1,2, \cdots, n\}$, one and only one of the following situations occurs:

(A) there are sequences $\left(i_{1}, i_{2}, \cdots, i_{k}\right)$ and $\left(j_{1}, j_{2}, \cdots, j_{l}\right)$ such that $B_{i_{1}, i_{2}, \cdots, i_{k}}$ $B_{j_{1}, j_{2}, \cdots, j_{l}}$ is an admissible product, $B_{i_{1}, i_{2}, \cdots, i_{k}} \nsim i, B_{j_{1}, j_{2}, \cdots, j_{l}} \sim i$ and $u \in T_{i_{1}, i_{2}, \cdots, i_{k}} I_{j_{1}, j_{2}, \cdots, j_{l}}^{i}$;

(B) there is a sequence $\left(i_{1}, i_{2}, \cdots, i_{k}\right)$ such that $B_{i_{1}, i_{2}, \cdots, i_{k}} \nsim i$ and $u \in T_{i_{1}, i_{2}, \cdots, i_{k}}$; 
(C) there is a sequence $\left(i_{1}, i_{2}, \cdots, i_{k}\right)$ such that $B_{i_{1}, i_{2}, \cdots, i_{k}} \sim i$ and $u \in I_{i_{1}, i_{2}, \cdots, i_{k}}^{i}$; (D) $u=1$.

Hence $L-\{1\}$ is the union of finitely many rational languages of type $T_{i_{1}, i_{2}, \cdots, i_{k}}$, $I_{i_{1}, i_{2}, \cdots, i_{k}}^{i}$ or $T_{i_{1}, i_{2}, \cdots, i_{k}} I_{j_{1}, j_{2}, \cdots, j_{l}}^{i}$, for appropriate choices of $\left(i_{1}, i_{2}, \cdots, i_{k}\right)$ and $\left(j_{1}, j_{2}, \cdots, j_{l}\right)$.

We will prove that, if each monoid $M_{i}$ is FGT, then $L_{a}$ is a finite union of rational languages and thus $L_{a}$ is rational.

\section{Case (A)}

Assume that $u \in T_{i_{1}, i_{2}, \cdots, i_{k}} I_{j_{1}, j_{2}, \cdots, j_{l}}$, for appropriate choices of $\left(i_{1}, i_{2}, \cdots, i_{k}\right)$ and $\left(j_{1}, j_{2}, \cdots, j_{l}\right)$. Assume also that $u=u_{1} u_{2} u_{3} u_{4}$, where

- $u_{1} \in X_{i_{1}, i_{2}, \cdots, i_{k}}$;

- $u_{2}=u_{2 i_{1}} u_{2 i_{2}} \cdots u_{2 i_{k}}$, where $u_{2 i_{m}} \in \overline{L_{i_{m}}}$, for every $m \in\{1,2, \cdots, k\}$;

- $u_{3}=u_{3 j_{1}} u_{3 j_{2}} \cdots u_{3 j_{l}}$, where $u_{3 j_{m}} \in \overline{L_{j_{m}}}$, for every $m \in\{1,2, \cdots, l\}$;

- $u_{4} \in Y_{j_{1}, j_{2}, \cdots, j_{l}}^{i}$.

Subcase (A1): $i \notin\left\{i_{1}, i_{2}, \cdots, i_{k}\right\}$.

Assume, without loss of generality, that $1 \leq j_{1}<\cdots<j_{m}<i<j_{m+1}<$ $\cdots<j_{l}$ and let $a^{\prime} \in \overline{L_{i}}$ be such that $a^{\prime} \phi_{i}=a \phi_{i}$. Since $\left(i_{1}, i_{2}, \cdots, i_{k}\right) \nsim i$ and $\left(j_{1}, j_{2}, \cdots, j_{l}\right) \sim i$ then $T_{i_{1}, i_{2}, \cdots, i_{k}} B_{j_{1}, j_{2}, \cdots, j_{m}, i, j_{m+1}, \cdots, j_{l}} Y_{j_{1}, j_{2}, \cdots, j_{l}}^{i}$ is still an admissible product and we have

- $v=u_{1} u_{2}\left(u_{3 j_{1}} \cdots u_{3 j_{m}} a^{\prime} u_{3 j_{m+1}} \cdots u_{3 j_{l}}\right) u_{4} \in L$

- $v \phi=(u a) \phi$

- $(u, v) \delta \in\left[\left(T_{i_{1}, i_{2}, \cdots, i_{k}} \overline{L_{j_{1}}} \cdots \overline{L_{j_{m}}}\right) \Delta\left(1, a^{\prime}\right) \delta\left(\overline{L_{j_{m+1}}} \cdots \overline{L_{j_{l}}} Y_{j_{1}, j_{2}, \cdots, j_{l}}^{i}\right) \Delta\right] \zeta$.

On the other hand, if $w \in\left[\left(T_{i_{1}, i_{2}, \cdots, i_{k}} \overline{L_{j_{1}}} \cdots \overline{L_{j_{m}}}\right) \Delta\left(1, a^{\prime}\right) \delta\left(\overline{L_{j_{m+1}}} \cdots \overline{L_{j_{l}}}\right.\right.$ $\left.\left.Y_{j_{1}, j_{2}, \cdots, j_{l}}^{i}\right) \Delta\right] \zeta$ then

- $w \pi_{1} \alpha \in T_{i_{1}, i_{2}, \cdots, i_{k}} I_{j_{1}, j_{2}, \cdots, j_{l}}^{i} \subseteq L$;

- $w \pi_{2} \alpha \in T_{i_{1}, i_{2}, \cdots, i_{k}} B_{j_{1}, j_{2}, \cdots, j_{m}, i, j_{m+1}, \cdots, j_{l}} Y_{j_{1}, j_{2}, \cdots, j_{l}}^{i} \subseteq L$;

- $\left(w \pi_{2} \alpha\right) \phi=\left(\left(w \pi_{1} \alpha\right) a\right) \phi$;

- $w=\left(w \pi_{1} \alpha, w \pi_{2} \alpha\right) \delta \in L_{a}$.

Then $\left[\left(T_{i_{1}, i_{2}, \cdots, i_{k}} \overline{L_{j_{1}}} \cdots \overline{L_{j_{m}}}\right) \Delta\left(1, a^{\prime}\right) \delta\left(\overline{L_{j_{m+1}}} \cdots \overline{L_{j_{l}}} Y_{j_{1}, j_{2}, \cdots, j_{l}}^{i}\right) \Delta\right] \zeta \subseteq L_{a}$ and by Lemma 3.2 and Proposition 6.3, $\left[\left(T_{i_{1}, i_{2}, \cdots, i_{k}} \overline{L_{j_{1}}} \cdots \overline{L_{j_{m}}}\right) \Delta\left(1, a^{\prime}\right) \delta\left(\overline{L_{j_{m+1}}} \cdots \overline{L_{j_{l}}}\right.\right.$ $\left.Y_{j_{1}, j_{2}, \cdots, j_{l}}^{i} \Delta\right] \zeta$ is rational.

Subcase (A2): $i=i_{m} \in\left\{i_{1}, i_{2}, \cdots, i_{k}\right\}$.

If $\left(u_{2 i} a\right) \phi_{i} \neq 1$, let $u_{2 i}^{\prime} \in \overline{L_{i}}$ be such that $u_{2 i}^{\prime} \phi_{i}=\left(u_{2 i} a\right) \phi_{i}$. Then we have $v=u_{1}\left(u_{2 i_{1}} \cdots u_{2 i_{m-1}} u_{2 i}^{\prime} u_{2 i_{m+1}} \cdots u_{2 i_{k}}\right) u_{3} u_{4} \in L$ and $v \phi=(u a) \phi$.

If $\left(u_{2 i} a\right) \phi_{i}=1$, since $\left(j_{1}, j_{2}, \cdots, j_{l}\right) \sim i$, the sequence $\left(i_{1}, i_{2}, \cdots, i_{m-1}\right.$, $\left.i_{m+1}, \cdots, i_{k}\right)$ is non-empty, $X_{i_{1}, i_{2}, \cdots, i_{k}} B_{i_{1}, i_{2}, \cdots, i_{m-1}, i_{m+1}, \cdots, i_{k}} I_{j_{1}, j_{2}, \cdots, j_{l}}^{i}$ is still an admissible product and $v=u_{1}\left(u_{2 i_{1}} \cdots u_{2 i_{m-1}} u_{2 i_{m+1}} \cdots u_{2 i_{k}}\right) u_{3} u_{4} \in L$. Hence, in both cases we have:

- $v \phi=(u a) \phi$

- $(u, v) \delta \in\left[\left(X_{i_{1}, i_{2}, \cdots, i_{k}} \overline{L_{i_{1}}} \cdots \overline{L_{i_{m-1}}}\right) \Delta\left(L_{i}\right)_{a}\left(\overline{L_{i_{m+1}}} \cdots \overline{L_{i_{k}}} I_{j_{1}, j_{2}, \cdots, j_{l}}^{i}\right) \Delta\right] \zeta$. 
On the other hand, if $w \in\left[\left(X_{i_{1}, i_{2}, \cdots, i_{k}} \overline{L_{i_{1}}} \cdots \overline{L_{i_{m-1}}}\right) \Delta\left(L_{i}\right)_{a}\left(\overline{L_{i_{m+1}}} \cdots \overline{{L_{i_{k}}}_{j_{1}}} I_{j_{2}, \cdots, j_{l}}^{i}\right)\right.$ $\Delta] \zeta$ then

- $w \pi_{1} \alpha, w \pi_{2} \alpha \in\left(T_{i_{1}, i_{2}, \cdots, i_{k}} I_{j_{1}, j_{2}, \cdots, j_{l}}^{i} \cup X_{i_{1}, i_{2}, \cdots, i_{k}} B_{i_{1}, i_{2}, \cdots, i_{m-1}, i_{m+1}, \cdots, i_{k}}\right.$ $I_{j_{1}, j_{2}, \cdots, j_{l}}^{i} \subseteq \subseteq L$

- $\left(w \pi_{2} \alpha\right) \phi=\left(\left(w \pi_{1} \alpha\right) a\right) \phi$

- $w=\left(w \pi_{1} \alpha, w \pi_{2} \alpha\right) \delta \in L_{a}$.

Hence we have, $\left[\left(X_{i_{1}, i_{2}, \cdots, i_{k}} \overline{L_{i_{1}}} \cdots \overline{L_{i_{m-1}}}\right) \Delta\left(L_{i}\right)_{a}\left(\overline{L_{i_{m+1}}} \cdots \overline{L_{i_{k}}} I_{j_{1}, j_{2}, \cdots, j_{1}}^{i}\right) \Delta\right] \zeta \subseteq$ $L_{a}$. Now, since $M_{i}$ is FGT, by Lemma $5.2, \Sigma\left(\left(L_{i}\right)_{a}\right)$ is finite and then, by Lemma 3.2 and Proposition 6.3, the language $\left[\left(X_{i_{1}, i_{2}, \cdots, i_{k}} \overline{L_{i_{1}}} \cdots \overline{L_{i_{m-1}}}\right) \Delta\left(L_{i}\right)_{a}\left(\overline{L_{i_{m+1}}} \cdots \overline{L_{i_{k}}}\right.\right.$ $\left.\left.I_{j_{1}, j_{2}, \cdots, j_{l}}^{i}\right) \Delta\right] \zeta$ is rational.

\section{Case (B)}

Assume that $u \in T_{i_{1}, i_{2}, \cdots, i_{k}}$ and $\left(i_{1}, i_{2}, \cdots, i_{k}\right) \nsim i$.

If $i \in\left\{i_{1}, i_{2}, \cdots, i_{k}\right\}$, this case corresponds to Subcase (A2), with language $I_{j_{1}, j_{2}, \cdots, j_{l}}^{i}$ replaced by the empty word.

If $i \notin\left\{i_{1}, i_{2}, \cdots, i_{k}\right\}$, let $a^{\prime} \in \overline{L_{i}}$ be such that $a^{\prime} \phi_{i}=a \phi_{i}$. Then $v=u a^{\prime} \in L$, $v \phi=(u a) \phi$ and $(u, v) \delta \in\left(T_{i_{1}, i_{2}, \cdots, i_{k}}\right) \Delta\left(1, a^{\prime}\right) \delta$. On the other hand $\left(T_{i_{1}, i_{2}, \cdots, i_{k}}\right)$ $\Delta\left(1, a^{\prime}\right) \delta$ is a rational language contained in $L_{a}$.

\section{Case $(\mathrm{C})$}

This case is Subcase (A1) with the language $T_{i_{1}, i_{2}, \cdots, i_{k}}$ replaced by the empty word.

\section{Case (D)}

If $u=1$ then $v=a^{\prime}$ is the single word of $L$ such that $v \phi=(u a) \phi=a \phi$. Hence $\left(1, a^{\prime}\right) \delta$ is a rational language contained in $L_{a}$.

Hence, $L_{a}$ is a finite union of rational languages and thus $L_{a}$ is rational. We have then proved the following result:

Theorem 6.4. Let $M=\Gamma\left(M_{1}, M_{2}, \cdots, M_{n}\right)$ be a graph product of monoids associated to a graph $\Gamma$. If, for every $i, M_{i}$ is an FGT automatic monoid then $M$ is automatic.

We will now establish a similar result for FGT $p$-automatic monoids:

Theorem 6.5. Let $M=\Gamma\left(M_{1}, M_{2}, \cdots, M_{n}\right)$ be a graph product of monoids associated to a graph $\Gamma$. If, for every $i, M_{i}$ is an FGT p-automatic monoid then $M$ is p-automatic.

Proof. By ([7], Cor. 5.4, Prop. 5.5 and Prop. 5.3) we may assume that there are p-automatic structures with uniqueness $L_{i}$ for $\phi_{i}: A_{i}^{+} \rightarrow M_{i}$ such that $1 \in L_{i}$, for every $i$. Furthermore, we may assume that the generating sets $A_{i}$ are disjoint. Let $A, \phi$ and $L$ defined as in the proof of Theorem 6.4. Then we already know that $L$ is an automatic structure with uniqueness for $\phi$ and thus we have to prove that $L_{=}^{\prime}=\{(u, v) \delta: u \in L, v \in \operatorname{Pref}(L)$ and $u \phi=v \phi\}$ is rational.

Given $v \in \operatorname{Pref}(L)$, one and only one of the following situations occurs:

(E) $v \in L$; 
(F) there is an independent sequence $\left(i_{1}, i_{2}, \cdots, i_{k}\right)$ and $m \in\{1,2, \cdots, k\}$ such that $v \in X_{i_{1}, i_{2}, \cdots, i_{k}} \overline{L_{i_{1}}} \overline{L_{i_{2}}} \cdots \overline{L_{i_{m-1}}}\left(\operatorname{Pref}\left(L_{i_{m}}\right)-\{1\}\right)$.

If (E) holds, the single word $u \in L$ such that $u \phi=v \phi$ is $v$ and then $(u, v) \delta \in L \Delta$. On the other hand, $L \Delta \subseteq L_{=}^{\prime}$ and $L \Delta$ is rational.

Assume now that (F) holds. Assume also that $v=v^{\prime} v_{i_{1}} v_{i_{2}} \cdots v_{i_{m-1}} v_{i_{m}}$ where $v^{\prime} \in X_{i_{1}, i_{2}, \cdots, i_{k}}, v_{i_{m}} \in \operatorname{Pref}\left(L_{i_{m}}\right)-\{1\}$ and $v_{i_{l}} \in \overline{L_{i_{l}}}$ for every $l \in\{1,2, \cdots, m-1\}$. Let $u_{i_{m}} \in L_{i_{m}}$ be such that $\left(u_{i_{m}}\right) \phi_{i_{m}}=\left(v_{i_{m}}\right) \phi_{i_{m}}$ and let $u=v^{\prime} v_{i_{1}} v_{i_{2}} \cdots v_{i_{m-1}} u_{i_{m}}$. Then

- $u \in X_{i_{1}, i_{2}, \cdots, i_{k}} \overline{L_{i_{1}}} \overline{L_{i_{2}}} \cdots \overline{L_{i_{m-1}}} L_{i_{m}} \subseteq L$;

- $u \phi=v \phi$

- $(u, v) \delta \in\left(X_{i_{1}, i_{2}, \cdots, i_{k}} \overline{L_{i_{1}}} \overline{L_{i_{2}}} \cdots \overline{L_{i_{m-1}}}\right) \Delta\left(L_{i_{m}}\right)_{=}^{\prime}$.

On the other hand, if $w \in\left(X_{i_{1}, i_{2}, \cdots, i_{k}} \overline{L_{i_{1}}} \overline{L_{i_{2}}} \cdots \overline{L_{i_{m-1}}}\right) \Delta\left(L_{i_{m}}\right)_{=}^{\prime}$ then we have

- $w \pi_{1} \alpha \in X_{i_{1}, i_{2}, \cdots, i_{k}} \overline{L_{i_{1}}} \overline{L_{i_{2}}} \cdots \overline{L_{i_{m-1}}} L_{i_{m}} \subseteq L$;

- $w \pi_{2} \alpha \in X_{i_{1}, i_{2}, \cdots, i_{k}} \overline{L_{i_{1}}} \frac{\overline{L_{i_{2}}}}{\cdots} \overline{L_{i_{m-1}}} \operatorname{Pref}\left(L_{i_{m}}\right) \subseteq \operatorname{Pref}(L)$;

- $\left(w \pi_{1} \alpha\right) \phi=\left(w \pi_{2} \alpha\right) \phi$;

- $w=\left(w \pi_{1} \alpha, w \pi_{2} \alpha\right) \delta \in L_{=}^{\prime}$.

Hence $\left(X_{i_{1}, i_{2}, \cdots, i_{k}} \overline{L_{i_{1}}} \overline{L_{i_{2}}} \cdots \overline{L_{i_{m-1}}}\right) \Delta\left(L_{i_{m}}\right)_{=}^{\prime} \subseteq L_{=}^{\prime}$ and by Proposition 6.3 is rational. Therefore $L_{=}^{\prime}$ is a finite union of rational languages and thus $L_{=}^{\prime}$ is rational.

\section{GRAPH PRODUCTS OF FGT MONOIDS}

In this section we will prove that the graph product of FGT monoids is FGT. For that we will need the following results:

Proposition 7.1 ([9], Prop. 7.1). Let $u \in S\left(X^{*}\right)$ be an irreducible word. If $v \in$ $S\left(X^{*}\right)$ is an irreducible word such that vu (uv) is congruent to the empty word then $v$ is $\Gamma$-equivalent to a product of left (right) inverses of the components of $u$, written by the opposite order.

Lemma 7.2 ([9], Lem. 14.1). Let $u, v \in S\left(X^{*}\right)$ be irreducible words. Then we may write $u \Leftrightarrow_{\Gamma} u_{3} u_{2} u_{1}$ and $v \Leftrightarrow_{\Gamma} v_{1} v_{2} v_{3}$ where

1. $u_{1} v_{1} \equiv 1$;

2. $u_{2}$ and $v_{2}$ are blocks of the same type;

3. $u_{3} S\left(u_{2} v_{2}\right) v_{3}$ is an irreducible word of $S\left(X^{*}\right)$ that is congruent to uv.

This lemma motivates the following definition: given an irreducible word $v \in$ $S\left(X^{*}\right)$ we say that $v_{1} v_{2} v_{3}$ is an $L$-form for $v$ if $v_{1}, v_{2}$ and $v_{3}$ are irreducible words of $S\left(X^{*}\right)$ such that

- $v \Leftrightarrow_{\Gamma} v_{1} v_{2} v_{3}$

- $v_{1}$ is left invertible;

- $v_{2}$ is a block of components. 
Theorem 7.3. Let $M=\Gamma\left(M_{1}, M_{2}, \cdots, M_{n}\right)$ be a graph product of monoids associated to a graph $\Gamma$. If, for every $i, M_{i}$ is FGT then $M$ is FGT.

Proof. Let $v$ be an irreducible word of $S\left(X^{*}\right)$. We want to prove that there is an integer $m_{v}$ such that, for every irreducible word $u$, there are at most $m_{v}$ non congruent irreducible words $x$ such that $x v$ and $u$ are congruent in $M$. By the preceding lemma, every irreducible word $x$, when multiplied by $v$, induces an Lform in $v$. Since there are finitely many L-forms for $v$ we just have to prove that, for every L-form of $v$ there is an integer $k$ such that, for every irreducible word $u$, there are at most $k$ non congruent irreducible words $x$, inducing that L-form for $v$, such that $x v$ and $u$ are congruent in $M$.

Let $v_{1} v_{2} v_{3}$ be an L-form for $v$. Since every monoid $M_{i}$ is FGT, every component $[t]$ of $v_{1} v_{2}$ has an associated integer $m_{t}$ and then let $k$ be the product of such integers. Let $u$ be an irreducible word of $S\left(X^{*}\right)$ and assume that $y$ and $z$ are irreducible words of $S\left(X^{*}\right)$, inducing the L-form $v_{1} v_{2} v_{3}$ for $v$, such that $y v$ and $z v$ are congruent to $u$. Then, by the preceding lemma, we may assume that $y=y_{3} y_{2} y_{1}$ and $z=z_{3} z_{2} z_{1}$, where

(i) $y_{1} v_{1}$ and $z_{1} v_{1}$ are congruent to 1 ;

(ii) $y_{2}$ and $z_{2}$ are blocks with the same type as $v_{2}$;

(iii) $y_{3} S\left(y_{2} v_{2}\right) v_{3}$ and $z_{3} S\left(z_{2} v_{2}\right) v_{3}$ are irreducible words of $S\left(X^{*}\right)$ that are congruent to $u$ in $M$.

From (i) and by Proposition 7.1, we may assume that $y_{1}$ and $z_{1}$ are products of left inverses of the components of $v_{1}$, written by the opposite order. Then, since every monoid $M_{i}$ is FGT, for every component $[t]$ of $v_{1}$, there are at most $m_{t}$ non congruent possibilities for the corresponding component of $y_{1}$ (or $z_{1}$ ).

Corresponding components of the blocks $S\left(y_{2} v_{2}\right)$ and $S\left(z_{2} v_{2}\right)$ are congruent to some component of $u$, in the respective monoid, and since every monoid $M_{i}$ is FGT, for every component $[t]$ of $v_{2}$ we have at most $m_{t}$ non congruent possibilities for the corresponding component of $y_{2}$ ( or $\left.z_{2}\right)$.

From (iii) and by Theorem 2.3, $y_{3} S\left(y_{2} v_{2}\right) v_{3}$ and $z_{3} S\left(z_{2} v_{2}\right) v_{3}$ are $\Gamma_{N}$-equivalent and thus, by Proposition $2.2, y_{3}$ and $z_{3}$ are $\Gamma_{N}$-equivalent.

Therefore, for every irreducible word $u$, we have at most $k$ non congruent possibilities for the irreducible word $y$ such that $y$ induces the L-form $v_{1} v_{2} v_{3}$ for $v$ and $y v$ is congruent to $u$.

\section{REFERENCES}

[1] C.M. Campbell, E.F. Robertson, N. Ruskuc and R.M. Thomas, Automatic Semigroups. Theoret. Comput. Sci. (to appear).

[2] A.J. Duncan, E.F. Robertson and N. Ruskuc, Automatic monoids and change of generators. Math. Proc. Cambridge Philos. Soc. 127 (1999) 403-409.

[3] E.R. Green, Graph Products of Groups, Ph.D. Thesis. The University of Leeds (1990).

[4] J.E. Hopcroft and J.D. Ullman, Introduction to Automata Theory, Languages and Computation. Addison-Wesley (1979). 
[5] S. Hermiller and J. Meier, Algorithms and Geometry for Graph Products of Groups. J. Algebra 171 (1995) 230-257.

[6] J.M. Howie, An Introduction to Semigroup Theory. Academic Press (1976).

[7] P.V. Silva and B. Steinberg, A Geometric Characterization of Automatic Monoids. Universidade do Porto (preprint).

[8] P.V. Silva and B. Steinberg, Extensions and Submonoids of Automatic Monoids. Universidade do Porto (preprint)

[9] A. Veloso da Costa, Graph Products of Monoids. Semigroup Forum 63 (2001) 247-277.

Communicated by Ch. Choffrut.

Received December, 2000. Accepted January, 2002.

To access this journal online: www.edpsciences.org 